\title{
Studies of a scheme for low emittance muon beam production from positrons on target
}

\author{
Francesco Collamati* ${ }^{*}$, Mario Antonelli ${ }^{b}$, Manuela Boscolo ${ }^{b}$, Marica Biagini ${ }^{b}$, Oscar \\ Blanco-Garcia $^{b}$, Alessandro Variola ${ }^{b}$, Alberto Bacci $^{c}$, Simone Liuzzo ${ }^{d}$, Pantaleo \\ Raimondi $^{d}$, Iryna Chaikovska ${ }^{e}$, Robert Cheab ${ }^{e}$, Matteo lafrati ${ }^{f}$, Lewis Keller ${ }^{g}$ et \\ Peter Sievers ${ }^{h}$ \\ ${ }^{a} I N F N$ - Rome, ${ }^{b} I N F N$ - LNF, INFN - Milan, ${ }^{d}$ ESRF - Grenoble, ${ }^{e}$ LAL - Orsay, ${ }^{f}$ ENEA - Frascati, \\ SLAC - Stanford, ${ }^{h}$ CERN - Geneve, \\ E-mail: francesco.collamati@romal.infn.it, \\ manuela.boscolo@lnf.infn.it, marica.biagini@lnf.infn.it, \\ oscar.blancoelnf.infn.it, alessandro.variola@lnf.infn.it, \\ alberto.bacci@mi.infn.it, simone.liuzzo@esrf.fr, \\ pantaleo.raimondi@esrf.fr, chaikovsdlal.in2p3.fr, \\ chehabelal.in2p3.fr, matteo.iafratidenea.it, \\ keller@slac.stanford.edu, peter.sievers@cern.ch,
}

\begin{abstract}
A new scheme to produce muon beams characterised by very low emittance, in such a way to avoid the need for cooling, using a positron beam of about $45 \mathrm{GeV}$ interacting on electrons on target is being studied by our group. This scheme is challenging and innovative, and needs a full design study to be developed. In particular, one of the novel topics to be investigated is the interaction between the positron beam stored in a low emittance ring with a thin target, to be inserted directly in the ring chamber to produce muons. Produced muons will then be immediately collected at the exit of the target and transported to two $\mu^{+}$and $\mu^{-}$accumulator rings. In this paper, after having highlighted the rational in designing a muon collider, we descrive in detail this new muon production scheme, discussing the simulation of the e+ beam interacting with the target, its degradation in the 6-D phase space and the optimisation of the e+ ring design mainly to maximise the energy acceptance.
\end{abstract}

The European Physical Society Conference on High Energy Physics

5-12 July

Venice, Italy

\footnotetext{
*Speaker.
} 


\section{Introduction}

Even if the idea of building a muon collider is not new at all, the first proposals date back to several decades ago, this hypothetical machine is gaining more and more interest today as a possible new generation of experiment, able to overtake the main limitations of actual colliders, and in particular of LHC. Even if the Large Hadron Collider has yet to deliver numerous years of data, due to the great effort needed to conceive and build such frontier machines it is already now the time to study how to carry on the research on fundamental particle physics in the next years.

In this context, design studies are now ongoing on a Future Circular Collider, to be build at CERN, with a circumference of about $100 \mathrm{~km}$ and unprecedented energies and luminosities for both hadron and lepton cases. However, even with respect to such huge and powerful machines, a muon collider would still retain some advantages that make it worth studying.

The majority of these advantages comes from the use of muons itself. In fact, being 200 times heavier than electrons, a beam of muons will suffer much less of Synchrotron Radiation emission, that is the limit of circular $e^{+} e^{-}$colliders, giving the possibility to reach much higher energies. Moreover, the lack of light emission also allows to obtain much smaller energy spread of the beam, resulting in a much higher energy resolution.

The advantages, however, are not only related to the accelerator, but also to the physics itself. In fact, focusing for example on the Higgs boson, we know that its coupling goes with $m^{2}$, meaning that we expect a much higher production of Higgs boson at a muon collider.

In spite of these advantages there are also some critical points that have hindered so far the design of a muon collider. First of all, muons decay in few $\mu$ s. That means that the whole chain, from generation to acceleration up to the interaction, must be performed very quickly. Moreover, the traditional muon production scheme leads inevitably to large emittance beams. In fact, muons are conventionally produced by colliding protons on target, and then exploiting the $\pi / k$ decays. Muons are thus produced with a variety of angles and energies, implying the necessity of beam cooling to lower the emittance of the beam.

In this paper, we describe a new scheme to produce muon beams characterised by intrinsic low emittance, in such a way to avoid the need for cooling.

\section{Direct muon production}

In this novel scheme, a primary beam of positrons interacts with a fixed target, the positron beam having a energy ( $45 \mathrm{GeV}$ ) just above the threshold for the reaction $e^{+} e^{-} \rightarrow \mu^{+} \mu^{-}$[1] [2]. The essential advantage of this "direct muon production" is that low emittance is possible. In fact, it happens that the angle between the 2 muons goes with $\sqrt{s}$, and is however small close to the threshold. Moreover, also the energy difference between the two muons produced is small just above the threshold, and thus is also the energy spread of the muon beam [referenza articolo]. As a result, exploiting direct muon production it is possible to create a muon beam that has already at creation small emittance, that in turns means that good luminosity is reachable with reduced muon fluxes, thus resulting in reduced backgrounds. Finally, also the losses from decays are reduced. In fact, the asymmetric scheme allows to obtain two already boosted muons, which will have higher lifetime and which are both possible to collect. 
However, on the other side with respect to such advantages, this novel scheme has its main limitation in the production rate, since the cross section for the process is about 3 orders of magnitude lower than the one for the conventional one ( $\mu b$ vs $m b)$.

Such a small cross section makes the choice of the target material crucial. In fact, the number of produced $\mu$ pairs $\left(N_{\mu \mu}\right)$ can be parametrised as:

$$
N_{\mu \mu}=N_{e^{+}} \rho_{e^{-}} L \sigma_{\left(e^{+} e^{-} \rightarrow \mu^{+} \mu^{-}\right)},
$$

where $N_{e^{+}}$is the number of positrons hitting the target, $\rho_{e^{-}}$is the electronic density of the target, $L$ is the length of the target, and $\sigma$ the cross section of the production process.

So, the criteria to follow to choose the target are basically 3 : first of all, to minimise the emittance a thin target is needed. In fact, having a thick target would mean that muons are produced all along its thickness, resulting in an increase in beam emittance. Secondly, the need to maximise the production rate requires a high density target. Lastly, the positron loss should be reduced as much as possible, since to rise the number of produced muons is it possible to conceive a recirculating scheme in which the positron beam interacts several times in the target. This last point would require a low $\mathrm{Z}$ target, in order to limit the effect of bremsstrahlung and Bhabha scattering, that would reduce the positron beam lifetime.

It is straightaway clear that these requirements are somehow conflicting with each other, and so a careful study relying on Monte Carlo simulation is being performed. A possible tradeoff between the conflicting requirements could be represented by not too heavy materials (like $\mathrm{Be}, \mathrm{C}$ and $\mathrm{Li}$ ) and not too thin targets.

The other fundamental aspect to be considered in order to maximise the production rate is the positron source, that has to be as intense as possible $\left(\sim 10^{18} e^{+} / s\right)$.

\section{Accelerator scheme}

The accelerator scheme currently proposed is shown in Fig. 1, and starts from a standard electron gun; these electrons collide with a conventional Heavy Target (HT) to produce $e^{+} e^{-}$pairs.

Then, an Adiabatic Matching Device is used to collect positrons, that are accelerated and injected in the main positron ring. At the current design stage, this is a $6.3 \mathrm{~km} 45 \mathrm{GeV}$ storage ring in which a target for muon production (T) is inserted, in such a way to make positrons collide with it several times. In such a configuration, muons are produced with an energy of $22 \mathrm{GeV}$ and a Lorentz $\gamma$ factor of about 200, implying that they will live about $500 \mu \mathrm{s}$. In correspondence of the production target there are two separate accumulation rings, followed by a fast acceleration that injects both muon beams in the actual collider.

A first set of parameters for the positron ring is shown in Fig. 2a, while a very preliminary set of parameters for a possible muon collider exploiting the proposed scheme is shown in Fig. $2 \mathrm{~b}$. However, what is worth to stress is that thanks to the very small emittance a luminosity comparable to the conventional one is possible with much lower muon fluxes.

\subsection{Positron ring optics scheme}

A complete optical design for the positron ring has been produced, consisting in a series of $200 \mathrm{~m}$ cells, with a total length of $6.3 \mathrm{~km}$. The beta functions and momentum acceptance for the 


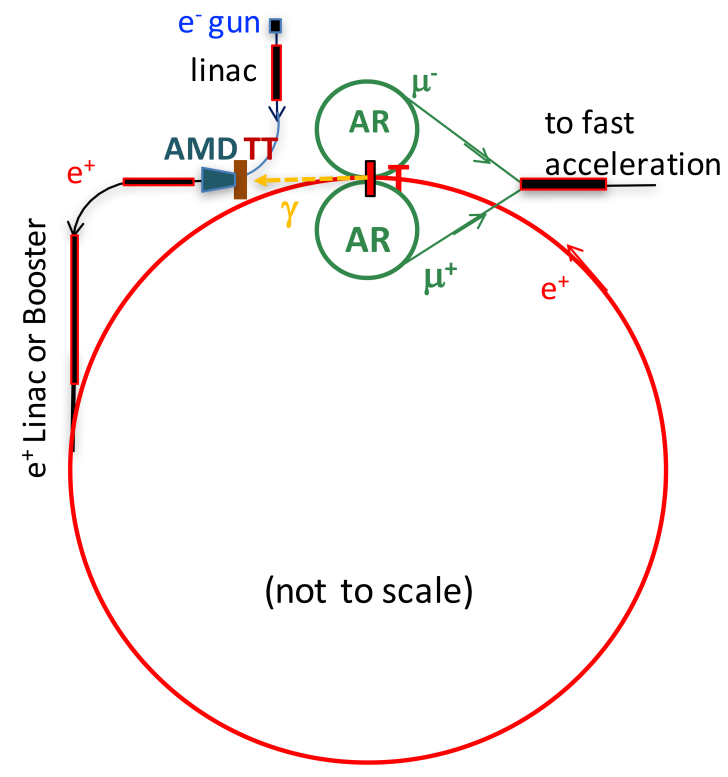

Figure 1: Actual design of the accelerator complex, as described in the text.

\begin{tabular}{|c|c|c|}
\hline $\begin{array}{c}\text { e+ ring parameter } \\
\text { Energy }\end{array}$ & unit & value \\
\hline $\begin{array}{c}\text { bunches } \\
\text { e+ bunch spacing } \\
\text { = Trev (AR) } \\
\text { Beam current } \\
\text { N(e+)/bunch }\end{array}$ & GeV & 6.3 \\
\hline Uo & ns & 100 \\
SR power & mA & 240 \\
\hline
\end{tabular}

\begin{tabular}{|c|c|c|}
\hline & & LEMC-6TeV \\
\hline Parameter & Units & \\
\hline LUMINOSITY/IP & $\mathrm{cm}^{-2} \mathrm{~s}^{-1}$ & $5.09 E+34$ \\
\hline Beam Energy & $\mathrm{GeV}$ & 3000 \\
\hline Hourglass reduction factor & & 1.000 \\
\hline Muon mass & $\mathrm{GeV}$ & 0.10566 \\
\hline Lifetime@prod & $\sec$ & $2.20 \mathrm{E}-06$ \\
\hline Lifetime & $\sec$ & 0.06 \\
\hline c*tau@prod & $\mathrm{m}$ & 658.00 \\
\hline$c *$ tau & $\mathrm{m}$ & $1.87 \mathrm{E}+07$ \\
\hline $1 /$ tau & $\mathrm{Hz}$ & $1.60 \mathrm{E}+01$ \\
\hline Circumference & $\mathrm{m}$ & 6000 \\
\hline Bending Field & $T$ & 15 \\
\hline Bending radius & $\mathrm{m}$ & 667 \\
\hline Magnetic rigidity & Tm & 10000 \\
\hline Gamma Lorentz factor & & 28392.96 \\
\hline $\mathrm{N}$ turns before decay & & 3113.76 \\
\hline$b_{x} @ \mathbb{P}$ & $\mathrm{m}$ & 0.0002 \\
\hline$b_{y} @ \mathbb{I P}$ & $\mathrm{m}$ & 0.0002 \\
\hline Beta ratio & & 1.0 \\
\hline Coupling (full current) & $\%$ & 100 \\
\hline Normalised Emittance $x$ & $\mathrm{~m}$ & $4.00 \mathrm{E}-08$ \\
\hline Emittance $\mathrm{x}$ & $\mathrm{m}$ & $1.41 \mathrm{E}-12$ \\
\hline Emittance $y$ & $\mathrm{~m}$ & $1.41 \mathrm{E}-12$ \\
\hline Emittance ratio & & 1.0 \\
\hline Bunch length (zero current) & $\mathrm{mm}$ & 0.1 \\
\hline Bunch length (full current) & $\mathrm{mm}$ & 0.1 \\
\hline Beam current & $\mathrm{mA}$ & 48 \\
\hline Revolution frequency & $\mathrm{Hz}$ & $5.00 E+04$ \\
\hline Revolution period & $\mathrm{s}$ & $2.00 \mathrm{E}-05$ \\
\hline Number of bunches & \# & 1 \\
\hline N. Particle/bunch & \# & $6.00 E+09$ \\
\hline Number of IP & \# & 1.00 \\
\hline$s_{x} @ \mathbb{I P}$ & micron & $1.68 \mathrm{E}-02$ \\
\hline sy@ IP & micron & $1.68 \mathrm{E}-02$ \\
\hline$S_{x^{\prime}} @ \mathbb{I P}$ & $\mathrm{rad}$ & $8.39 \mathrm{E}-05$ \\
\hline Sy' @IP & $\mathrm{rad}$ & $8.39 \mathrm{E}-05$ \\
\hline
\end{tabular}

Figure 2: On the left (a): set of parameters for the positron ring. On the right (b): set of preliminary parameters for a possible muon collider exploiting the described scheme. 
cell are shown in Fig. 3, and are being studied both with MADX-PTC and Accelerator Toolbox, finding good agreement between the two codes. Some more details on the machine are reported in the table in Fig. 3.

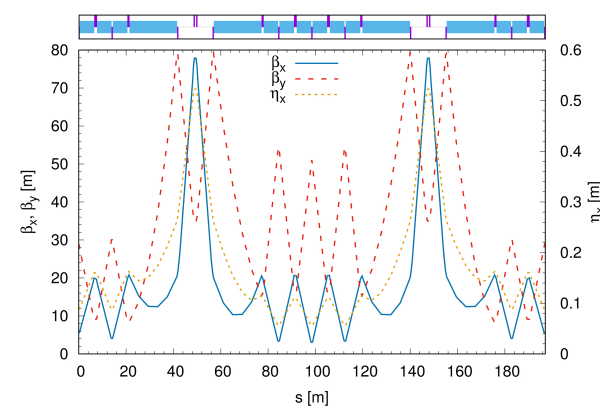

\begin{tabular}{lll}
\hline \hline Parameter & Units & \\
Energy & $\mathrm{GeV}$ & 45 \\
Circumference & $\mathrm{m}$ & 6300 \\
Coupling(full current) & $\%$ & 1 \\
Emittance $\mathrm{x}$ & $\mathrm{m}$ & $5.73 \times 10^{-9}$ \\
Emittance y & $\mathrm{m}$ & $5.73 \times 10^{-11}$ \\
Bunch length & $\mathrm{mm}$ & 3 \\
Beam current & $\mathrm{mA}$ & 240 \\
RF frequency & $\mathrm{MHz}$ & 500 \\
RF voltage & $\mathrm{GV}$ & 1.15 \\
Harmonic number & $\#$ & 10508 \\
Number of bunches & $\#$ & 100 \\
N. particles/bunch & \# & $3.15 \times 10^{11}$ \\
Synchrotron tune & & 0.068 \\
Transverse damping time & turns & 175 \\
Longitudinal damping time & turns & 87.5 \\
Energy loss/turn & $\mathrm{GeV}$ & 0.511 \\
Momentum compaction & & $1.1 \times 10^{-4}$ \\
RF acceptance & $\%$ & \pm 7.2 \\
Energy spread & $\mathrm{dE} / \mathrm{E}$ & $1 \times 10^{-3}$ \\
SR power & $\mathrm{MW}$ & 120 \\
\hline \hline
\end{tabular}

Figure 3: Twiss functions (a) and momentum acceptance (b) of the positron ring cells, studied with MAD-X PTC and Accelerator Toolbox, and a table (c) with some parameters of the lattice.

\subsection{Low $\beta$ interaction region insertion}

The ideal goal for this machine would be to have a zero dispersion and low beta Interaction Region in which to place the muon production target, in order to minimise the degradation produced by the target and maximise the muon production. For the moment, the optimisation of the lattice allowed to reach these values at the target position:

$$
\beta_{x}=1.6 m ; \beta_{y}=1.7 m ; D_{x}=5.4 m m .
$$

The dynamic and momentum aperture corresponding to this values are shown in Fig. 4, where it is clear the worsening effect of adding the Insertion Region in the cell. However, also in this field further optimisation are underway, in particular attempting to match the transverse minimum beam size with constraints of target thermo-mechanical stress, that put some limits on the size of the beam impinging on it. Also dynamic and momentum aperture can still be optimised.

\section{Multi turn simulation studies}

Due to the peculiarities of the proposed machine, with multiple interactions of the primary beam within the target, a dedicated multi turn simulation scheme has been set up, in order to follow the evolution of the beam and to optimise its parameters. The simulation starts with the initial $6 \mathrm{D}$ 

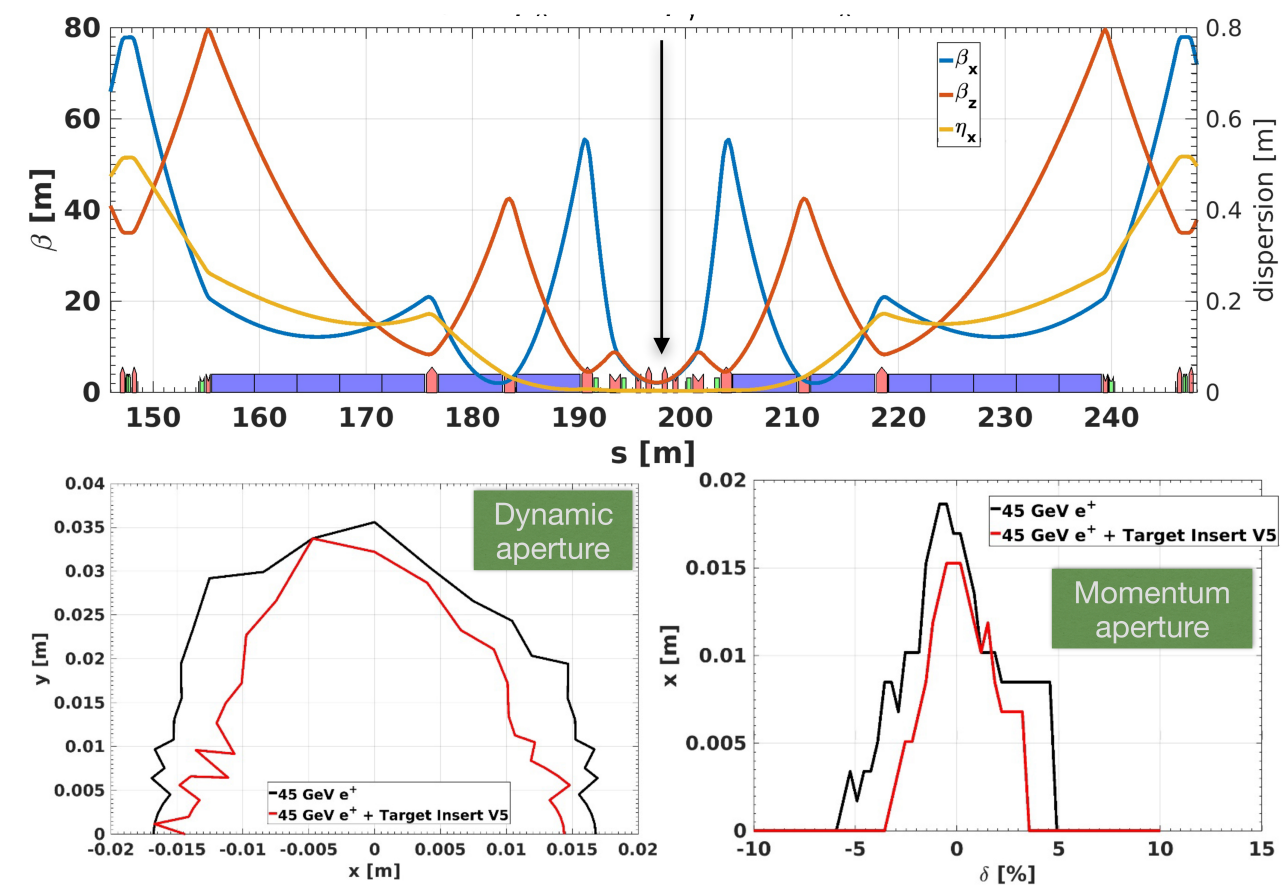

Figure 4: Top: Beta functions and Dispersion when adding the target to te cell. Bottom: dynamic (left) and momentum (aperture) of the cell when inserting the target.

beam distribution, taken from the equilibrium emittances. This distribution is tracked for a whole turn up to the target using both Accelerator Toolbox and MAD-X PTC for comparison. Then, the behaviour of the positrons inside the target is simulated by means of FLUKA and GEANT4 Monte Carlo codes, which exit distribution is sent back to the tracking program until the next interaction in the target.

So, at each pass through the target the positron beam undergoes two processes. Firstly, it gets an angular kick due to Multiple Scattering, leading to an increase in the emittance. Secondly, positrons loose energy due to bremsstrahlung, and thus the role of momentum acceptance of the positron ring becomes crucial.

By means of this multi turn simulation it is also possible for example to study the variation of the positron beam lifetime as a function of the target thickness and material, and also to evaluate which is the process that dominates the loss, that turns out to be radiative loss in the target, rather than Multiple Scattering, as shown in Fig. 5.

Fig. 6 shows the phase space plots for several stages of the multi turn simulation (first turn, turn n. 35 and superimposition of the first 35 turns) for the case of $3 \mathrm{~mm}$ Beryllium target.

Moreover, this multi turn simulation allows to evaluate the increase in beam size as a function of turns, also factorising the contribution coming from bremsstrahlung and Multiple Scattering to this emittance increase. As shown in Fig. 6, it turns out that in the current machine design the dominating contribution is given by bremsstrahlung, that however is expected to become almost negligible once a 0 dispersion interaction region will be available. 

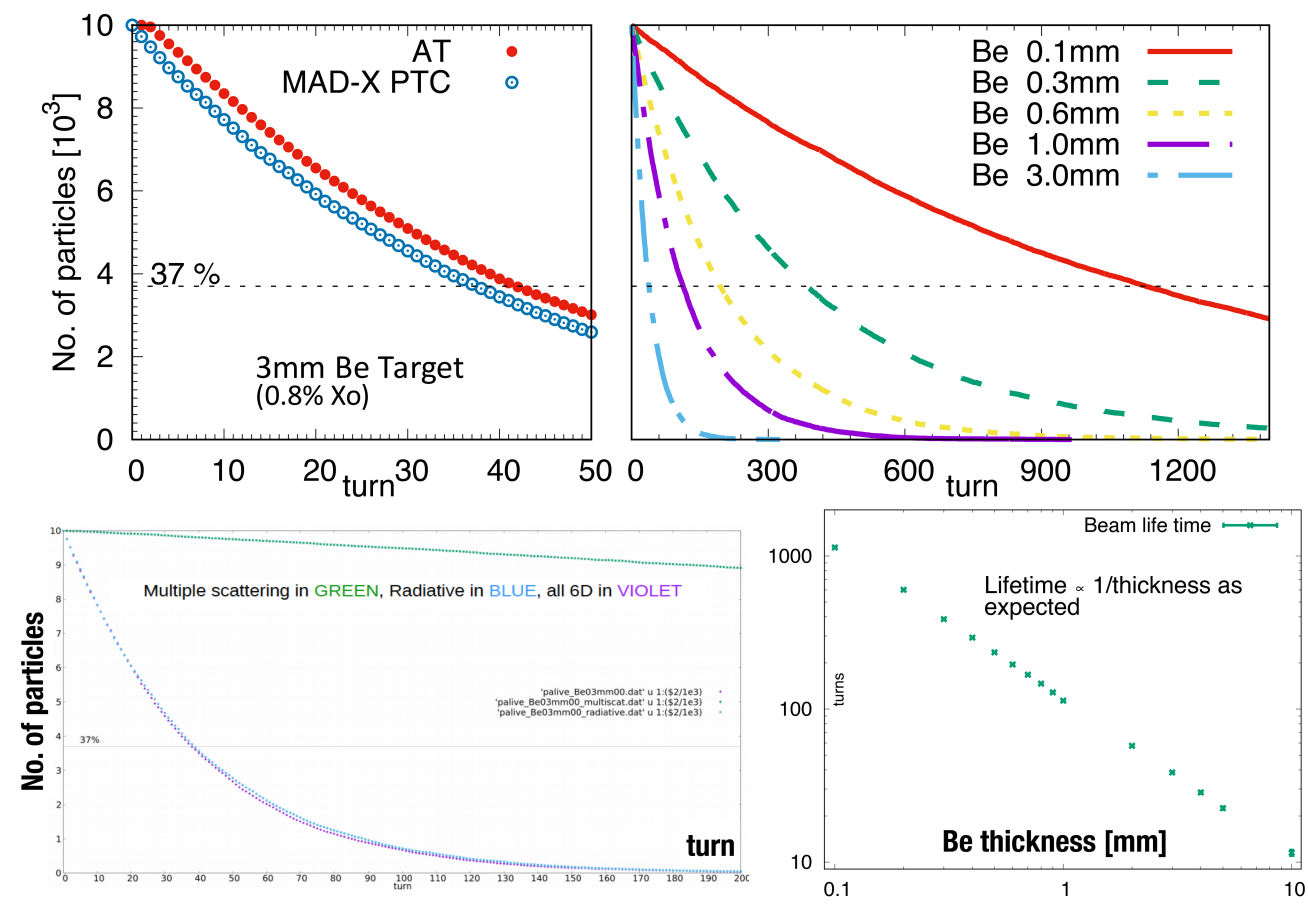

Figure 5: Top: fraction of particles surviving as a function of number of turns for several thicknesses of Beryllium target (left: zoom for $3 \mathrm{~mm}$ Be case). Bottom left: contribution to particle losses for the two processes. Bottom right: beam lifetime as a function of target thickness.

\section{Conclusion}

In this paper, a novel approach to muon production was described, that could allow the design of a muon collider characterised by very low emittance without the need of cooling. To overcome the main limitation of this new production scheme, that is the low rate due to the small cross section of the process of interest, it is crucial to optimise the target choice, in such a way to maximise the number of muons created, resulting in the need to sustain a high radiation load.

A first design of the low emittance positron ring has been developed and presented in this paper, with preliminary studies of beam dynamics, also exploiting a multi turn simulation that has been specifically developed joining tracking codes like MAD-X PTC or Accelerator Toolbox with Monte Carlo codes like FLUKA and GEANT4.

Even if there is still plenty of room for optimisation in several aspects, from the machine to the target to the positron source needed, the first results shown in this paper seems to be promising. 

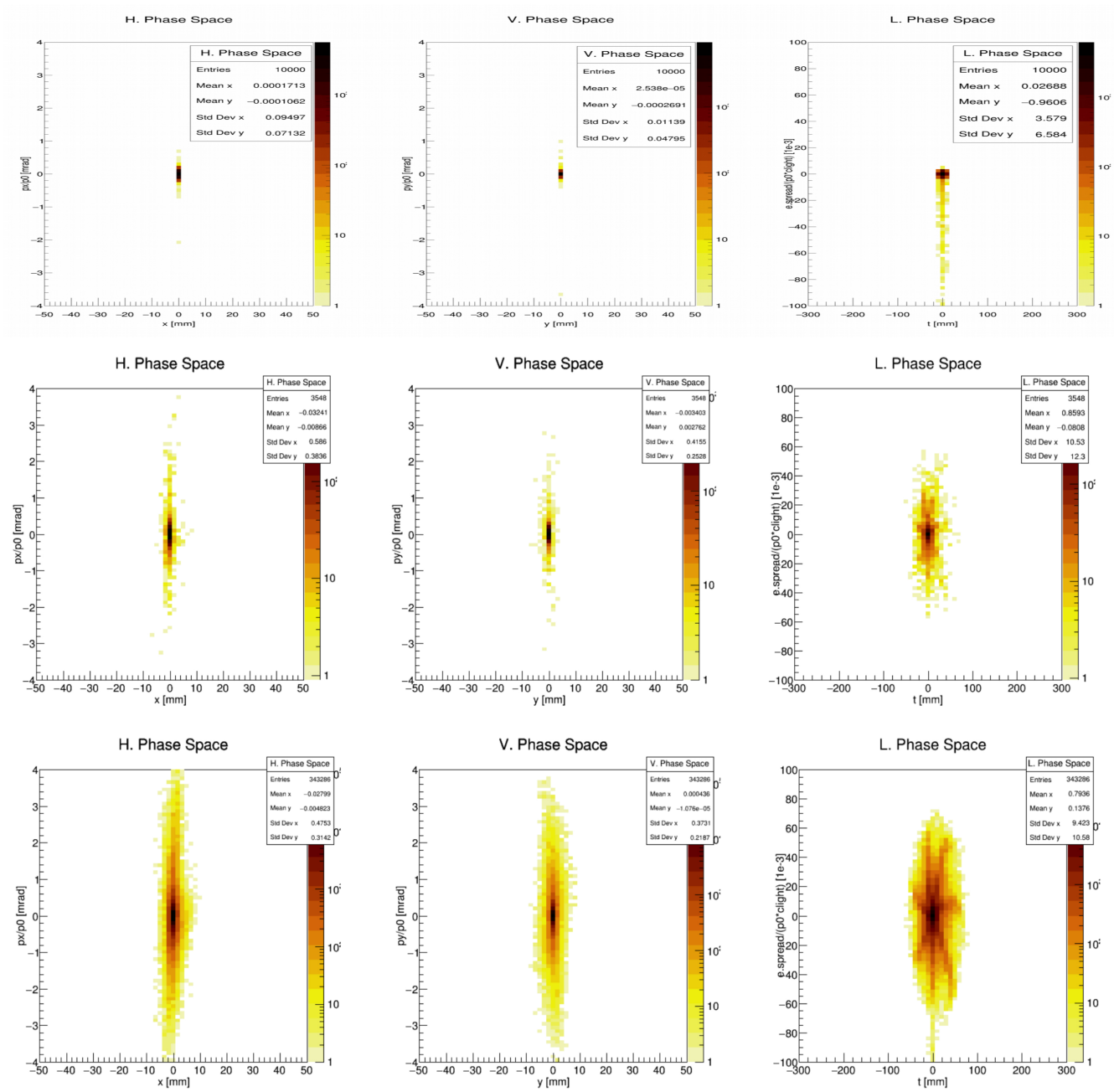

Figure 6: Phase space plots for the first turn of the simulation (top), turn n. 35 (middle) and the superimposition of the first 35 turns (bottom).

\section{References}

[1] M. Antonelli, M. Boscolo, R. Di Nardo, P. Raimondi, Novel proposal for a low emittance muon beam using positron beam on target, Nuclear Instruments and Methods in Physics Research A 807 (2016) 101-107

[2] M. Antonelli, M. Biagini, M. Boscolo, A. Variola, P. Raimondi, G. Cavoto, E. Bagli, Very low emittance muon beam using positron beam on target, Proceeding of IPAC2016 - Busan, Corea (TUPMY001) 